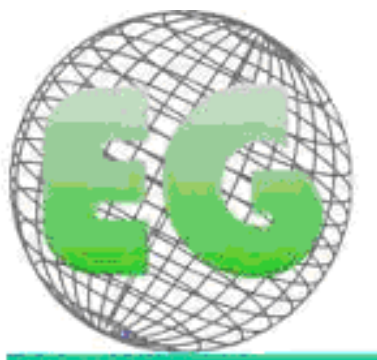

15SN $1696-6$ *⿻

$N^{\circ} 19$
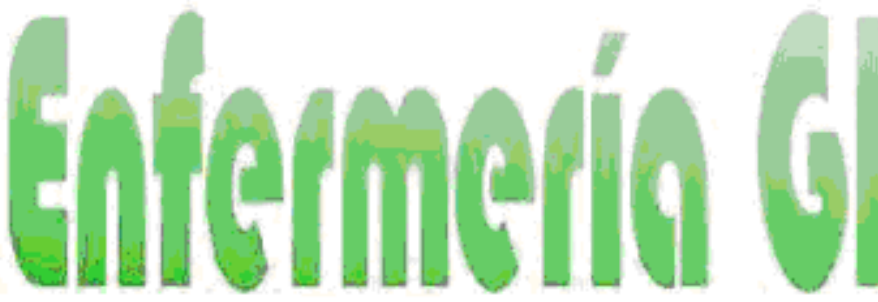

Revista electrônica cuatrimestral de Enfermeria

Junio 2010

\title{
LA CONCEPCIÓN DEL ENFERMERO SOBRE GERENCIAMIENTO DEL COSTO HOSPITALARIO
}

A CONCEPÇÃO DO ENFERMEIRO SOBRE GERENCIAMENTO DO CUSTO HOSPITALAR

${ }^{*}$ Da Silva, DG, ${ }^{* *}$ Dos Reis, LB, ${ }^{* * *}$ Marinho Chrizóstimo, M., ${ }^{* * * * C a r v a l h o ~ A l v e s, ~}$ EM.

*Enfermeira. **Enfermeira. Mestre em Administração. ***Enfermeira. Mestre em Educação. ****Mestre em Enfermagem. Escola de Enfermagem Aurora de Afonso Costa da Universidade Federal Fluminense. Brasil.

Palabras-clave: Gerencia; Costo hospitalario; Enfermero.

Palavras-chave: Gerenciamento; Custo hospitalar; Enfermeiro.

\section{RESUMEN}

Estudio trata del conocimiento que el enfermero tiene sobre la gerencia de costo hospitalario por el enfermero. Objetiva identificar el papel del enfermero en la gerencia de costo hospitalario y su concepción de este proceso; verificar la tendencia de las acciones del enfermero en esta gerencia; identificar las facilidades encontradas por los enfermeros en la implantación del control de costos. Estudio de abordaje cualitativo; se usó el cuestionario como instrumento para colecta de datos. Los participantes son enfermeros del Hospital Universitario Antonio Pedro (Niterói/RJ). Se utilizó el método de análisis de contenido con categorización de los temas. Se concluyó que la concepción del enfermero sobre la gerencia del costo hospitalario es muy restringida y desestructurada. A medida que los enfermeros perfeccionen y comprendan el proceso de control de costo, sus acciones en la gerencia de este proceso, elevará el patrón de calidad de la asistencia.

\section{RESUMO}

Estudo trata do conhecimento que o enfermeiro tem sobre o gerenciamento de custo hospitalar pelo enfermeiro. Objetiva identificar o papel do enfermeiro no gerenciamento de custo hospitalar e sua concepção deste processo; verificar a tendência das ações do enfermeiro neste gerenciamento; identificar as facilidades encontradas pelos enfermeiros na implantação do controle de custos. Pesquisa de abordagem qualitativa; usou o questionário como instrumento para coleta de dados. Os participantes são enfermeiros do Hospital Universitário Antonio Pedro (Niterói/RJ). Utilizou-se o método de análise de conteúdo com categorização dos temas. Concluiu-se que a concepção do enfermeiro sobre o gerenciamento do custo hospitalar é muito restrita e desestruturada. À medida 
que os enfermeiros aperfeiçoarem e compreenderem o processo de controle de custo, suas ações no gerenciamento deste processo, elevará o padrão de qualidade da assistência.

\section{INTRODUCCIÓN}

Actualmente el Sistema Único de Salud (SUS), legitimado por la Constitución de 1988, aún se encuentra en construcción. La participación de la sociedad; el compromiso de los actores sociales que representan gestión, comunidad, órgano formador de los profesionales de salud y la implementación de la acción reglamentada legalmente por el Poder Público haría que que el SUS se tornase realidad.

Por eso, la atención a la salud, con alto padrón de calidad, es un derecho a ser conquistado. Uno de los desafíos de la política pública de salud, para el gestor, es ofertar atención de calidad a la población respetando preceptos preconizados por el SUS, la universalidad, igualdad y equidad.

Para eso se sabe que la gerencia de la asistencia en salud está constituida en salud por las categorías administrativa y asistencial. Y que el concepto de administrar consiste en "tomar decisiones sobre los objetivos a ser alcanzados por la organización y sobre la utilización de sus recursos. Esas decisiones se clasifican en cuatro tipos principales: planeamiento, organización, dirección y control"1-3

El proceso administrativo en la salud tiene como principal función a la asistencia de calidad que proporcione satisfacción del ciudadano, a través de proyectos definidos y estructurados por la institución y que puedan ser alcanzados mediante el trabajo de los profesionales de la salud.

"Gerencia es el arte de pensar, de decidir y de actuar; es el arte de hacer acontecer, de obtener resultados. Resultados que pueden ser definidos, previstos, analizados y evaluados, mas que tienen de ser alcanzados a través de las personas y en una interacción humana constante"3

La asistencia multiprofesional, en la salud, posee como centro el paciente, y es orientada por el planeamiento, por la dirección, y evaluación de actividades desarrolladas por el personal auxiliar y la supervisión, todos atendiendo a las necesidades presentadas por los pacientes. Abarca la coordinación de las actividades desarrolladas con los clientes de su unidad por personas de otros servicios ${ }^{4}$. Para el desarrollo de las acciones asistenciales y en medio de las condiciones socio-económicas en que el país está inserto, resultante de la política de gobierno vigente, saber administrar los recursos disponibles ha sido la principal actividad de las organizaciones, sean familiares o empresariales como la institución hospitalaria. Por lo tanto, para comprensión de esta gestión uno de los puntos a ser considerado es el perfil de la asistencia ofrecida por el enfermero y la financiación de las acciones de cada unidad dentro del Sistema.

Administrar costos, dentro de presupuestos restringidos, es mantener equilibrio entre gastos, costos y recetas, garantizando la permanencia de la organización, pudiendo visualizar la unidad hospitalaria como una empresa ${ }^{5}$. Los costos pueden ser clasificados en indirectos y directos, fijos y variables. "Los costos indirectos son comunes a diversos procedimientos 0 servicios, no pudiendo ser atribuidos, exclusivamente, a un sector o producto. Su apropiación se hace por medio de criterios o fórmulas de rateo, basados en algún factor volumétrico para ser debitados a un producto o servicio" 5 . 
"Los costos directos son gastos efectuados directamente a la población o atención". Los costos fijos "son los que permanecen constantes dentro de cierto intervalo de tiempo, independientes de las variaciones ocurridas en el volumen de producción y vendas durante ese período". Y, costos variables "son aquellos cuyo valor total aumenta o disminuye directa y proporcionalmente con las flotaciones ocurridas en la producción y vendas"6-7.

En los ambientes hospitalarios, la contabilidad de costos es un instrumento gerencial importante para la determinación, control y análisis de los gastos (costos y gastos), permitiendo el confronto de eses costos y gastos con los precios de los procedimientos realizados, que son definidos por el mercado, compuesto de compañía de seguros, cooperativas de salud, gobierno y otros ${ }^{8}$.

El control de costo hospitalario es, aún, un proceso desvalorizado por muchos profesionales de la equipo de la salud dificultando la implementación y perfeccionamiento de la administración de costos.

La administración de calidad tiene como principio atender perfectamente, de forma confiable, de forma accesible, de forma segura y en el tiempo cierto las necesidades del cliente ${ }^{9}$.Por fin el proceso de administración de costo tiene el propósito de calificar, gradualmente y de forma constante, el trabajo del equipo de enfermería. La ejecución responsable de esta atribución optimiza la asistencia en salud, a medida que dirige, de forma pertinente, los recursos disponibles y prioriza el control de los gastos a través de actividades de supervisión desarrolladas por el enfermero.

En este contexto, se pretende compatibilizar la calidad del servicio con el gasto, presupuesto, gasto y el financiero de la institución, para que se construyan prácticas humanizadas, eficientes y eficaces en el proceso de trabajo del equipo de salud, gestión del sistema hospitalario y la administración de los recursos financieros sean estos oriundos del sistema público o privado, evitando gastos exorbitantes y aplicación inadecuada del recurso monetario disponible.

El interés por el desarrollo de este estudio surgió a través de la vivencia de profesionales ligados directa o indirectamente a la ejecución de la asistencia en el Sistema Público de Salud donde lidian diariamente con la carencia de recursos materiales, humanos y financieros. Las cuestiones que nortearon la referida pesquisa fueron:

- ¿Cuál es el conocimiento del enfermero sobre el costo de la asistencia prestada al paciente?

- ¿A quién compete controlar los costos dentro de una unidad hospitalaria?

- ¿Existe un control de stock para artículos usados y almacenados en las unidades?

- ¿Cuáles son las actividades del enfermero que desarrolla, participa y/o colabora para el control de los costos de la asistencia?

- ¿Cuáles acciones deberían ser desarrolladas para facilitar y/o colaborar con el control de los costos de la asistencia?

Así este estudio tuvo como objetivos: identificar el papel del enfermero en el proceso de gerencia de costo hospitalario y su concepción sobre la gerencia del costo de la asistencia al paciente; verificar la tendencia de las acciones del enfermero en la gerencia de costo hospitalario e identificar las facilidades encontradas por los enfermeros gestores en la implantación del control de costos. 


\section{METODOLOGÍA}

Se trata de una pesquisa de campo con abordaje cualitativo en la cual la interacción entre el investigador y el sujeto es esencial ${ }^{10}$. Para el desarrollo del estudio fueron atendidos los requisitos de la Resolución 196/96. Los sujetos pesquisados fueron doce enfermeros y tres enfermeros gerentes del Hospital Universitário Antonio Pedro de la Universidade Federal Fluminense.

La colecta de datos ocurrió en el primer semestre de 2006. Para la colecta fue utilizado el cuestionario, como instrumento, lo cual es un medio de obtener respuestas a las preguntas que el propio informante rellena; contiene un conjunto de cuestiones, todas lógicamente relacionadas con un problema central ${ }^{11}$. Es posible afirmar que hubo precategorización en la elaboración del cuestionario. Fue explicado al enfermero, antes de la colecta de datos, los objetivos de la pesquisa así como la posibilidad de, en cualquier momento, solicitar su abandono. Después de los esclarecimientos necesarios, el participante firmó el Término de Consentimiento Libre y Esclarecido descrito en la Resolución 196/96 del Consejo Nacional de Pesquisa.

Para evaluación de las informaciones fue utilizada una aproximación al método de análisis de contenido que apunta a hacer evidentes y significativamente plausibles la corroboración lógica de los elementos ocultos del lenguaje humano, allende organizar y descubrir el significado original de sus elementos manifiestos ${ }^{12}$. En la discusión de los datos, las informaciones obtenidas con el cuestionario fueron transcritas utilizando nombres ficticios de flores, para garantizar el anonimato de los enfermeros.

\section{ANÁLISIS DE LOS RESULTADOS}

Después de la colecta de los datos para análisis del contenido, fue utilizada la categorización de los temas conforme preestablecidos en el cuestionario: Conocimiento; Responsabilidad; Control del Material bajo la guarda del Enfermero; Acciones para el Control de los Costos y Acciones que deberían ser desarrolladas para el Control de los Costos de la Asistencia.

\section{$\underline{\text { Conocimiento }}$}

Se preguntó al enfermero cuál era su conocimiento sobre el costo de la asistencia prestada al paciente. De entre los 15 enfermeros entrevistados 6,6\% definieron costo como la fuerza de trabajo envuelta en las acciones del cuidar. Otros $6,6 \%$ describieron elementos que pueden ser encuadrados como costos directos e indirectos. Aproximadamente 13\% no supieron responder. Un total de $33,3 \%$ participantes presentaron varias opiniones, tales como: "solo sabía lo que era caro y lo que era barato"; respuestas evasivas que demostraban conocimiento muy restringido sobre el tema cuestionado. Finalizando un $40 \%$ que definieron como siendo principalmente el gasto con material.

El valor del material y calidad de los mismos, la relación entre ellos y la forma que utilizamos este material interfiere en la adquisición de otros. Orquídea

Es todo material permanente, de consumo y las actividades asistenciales prestadas al cliente. Margarida 


\section{Responsabilidad}

El segundo cuestionamiento fue: ¿de quién es la responsabilidad y cuáles son los profesionales que deben estar implicados en el control de costos?

Hubo $6,6 \%$ de los enfermeros que respondieron que el enfermero; $6,6 \%$ relataron ser del enfermero, del médico y de la administración; 6,6\% dijeron ser del médico, del enfermero y su equipo y $80 \%$ afirmaron que todos son responsables.

Todos deben estar implicados, desde el personal que providencia la compra, hasta el profesional que utiliza el material. Violeta

Todo equipo multidisciplinar; muchos problemas asistenciales podrían ser minimizados si todo equipo tuviese consciencia de los costos asistenciales. Margarida

\section{Control del Material bajo la guarda del Enfermero}

Fue interrogado: ¿existe control de stock para ítems utilizados y almacenados en las unidades?

De la totalidad de los enfermeros que participaron de la pesquisa, 6,6\% dijeron que existe un control que está en fase inicial. Otros $13 \%$ afirmaron que, generalmente, no hay control de estoque. Que existe un control fue relatado por $20 \%$ de los entrevistados, mas no explicaron cómo es ejecutado. La existencia del control fue informada por $26,6 \%$, sin embargo dicen que es realizada de forma inadecuada. De entre los respondientes 33\% dijeron que existe un control del material y describieron que es desarrollada a través de la verificación del estoque de la unidad y solicitación al depósito.

Sí. Verifico el consumo gasto en el estoque y solicito la reposición. Hortência

El almacenamiento en la unidad ocurre por una semana y algunos ítems por más tiempo. El controle de estoque se intentó, sin embargo no permaneció debido a la no adhesión de otros enfermeros de la unidad. Orquídea

\section{Acciones para el Control de los Costos}

Para discutir esta categoría fue cuestionado sobre las acciones que son desarrolladas, que participan y/o colaboran para el control de los costos de la asistencia.

Cerca de $6 \%$ de los profesionales relataron que promueven la solicitación de material y la utilización del material con celo; 6,6\% afirmaron que hacen la utilización del material con celo; 6,6\% relataron responder parecer técnico, participar de pesquisas y promover orientación de las equipos; 6,6\% dijeron participar de la asistencia y controlar el material de la unidad; 6,6\% afirmaron que hacen el control de la producción; 6,6\% relataron no desarrollar ninguna acción; $13,3 \%$ dijeron solo orientar los equipos y $46 \%$ afirmaron responder parecer técnico y desarrollar el control del material.

Participo, haciendo control de estoque de materiales utilizados en los procedimientos. Rosa

En verdad, por tratarse de una institución pública las acciones son más en el sentido de controlar el material (consumo) existente, que de los costos en síu Cravo 


\section{Acciones que deberían ser desarrolladas para el Control de los Costos de la Asistencia}

Finalizando las categorías, fue preguntado: cuales acciones que el enfermero acredita que deberían ser desarrolladas para facilitar y/o colaborar con el control de los costos de la asistencia. En esta pregunta las respuestas fueron cuantificadas conforme el número de veces en que los ítems fueron citados, diferentemente de las categorías anteriores donde hube la cuantificación de los enfermeros.

La elaboración de protocolos fue citada dos veces; el Parecer Técnico y el Proceso Licitatorio fueron citados dos veces; evitar el desperdicio, tres veces; disponibilidad de material de calidad fue citado tres veces; las actividades de Educación Continuada fueron referidas cinco veces y el control de material seis veces.

Educación continuada en todos los niveles con palestras, vídeos, propagandas, etc. Copo-de-leche

Sensibilizar los funcionarios para la importancia de los gastos hospitalarios, envolverlos en la gerencia para que los mismos perciban como ellos son parte fundamental del proceso de gastos. Proceso de Licitación (Pregão) que genera concurrencia entre los proveedores, disminuyendo los precios de los productos. Azaléia

\section{CONSIDERACIONES FINALES}

La carencia de recursos materiales necesarios a la asistencia refleja el contexto filosófico, político, económico y social vigente en el mundo globalizado. Somos regidos por un sistema económico capitalista, de crecimiento continuado de la tecnología lo que se que refleja en el aumento de los gastos. Siendo necesaria la utilización de los recursos financieros con consciencia y responsabilidad para evitar el uso indebido de los recursos.

Con la carencia de recursos financieros en el sector hospitalario es necesario administrar adecuadamente el proceso de la asistencia, a través del ejercicio efectivo del enfermero en el control de los costos dentro de la unidad que administra.

En el transcurrir de la pesquisa quedó evidenciada que la concepción del enfermero sobre la gerencia del costo de la asistencia al paciente es muy limitada por el desconocimiento del proceso de control de costos, su relevancia, y probablemente por el hecho de la institución de no priorizar esta práctica por los enfermeros.

Se constató que el papel del enfermero en el proceso de administración de costo hospitalario se restringe, principalmente, al control de los materiales de la unidad; ítem que consideran como lo más importante en el costo de la asistencia. Se evidenció en el análisis, que la mayor parte de los respondientes tiene el concepto claro de que todos los profesionales son responsables por el control de los costos. Los enfermeros afirmaron, que realizan algún control en el estoque de materiales. Todavía, al describir ese proceso se puede percibir que los mismos desconocen las etapas de previsión, provisión, organización y control que compone el proceso de control de materiales de una determinada unidad.

La administración de material en las unidades de enfermería abarca las funciones de previsión, provisión, organización y control. Con esto, el equipo de enfermería encuentra 
dificultad en optimizar el control de los gastos y proporcionar una asistencia con alto padrón de calidad.

Entretanto, se verificó que los enfermeros comprenden algunas de las actividades que deben ser desarrolladas para facilitar sus acciones en el control de los costos de la asistencia: respuesta a los Pareceres Técnicos que integran el Proceso Licitatorio; evitar el desperdicio de materiales; disponibilidad de materiales de calidad y actuación efectiva del servició de Educación Continuada los capacitaría para la administración del proceso de costo hospitalaria.

A medida que los enfermeros perfeccionan y comprenden el proceso de control de costos en su totalidad, sus acciones en el gerenciamento del costo hospitalario tienden a ampliarse.

Independientemente de que los hospitales sean públicos o privados es necesario que haya la provisión adecuada de los recursos materiales, humanos y financieros. Con esto las instituciones del sistema de salud han procurado ampliar y perfeccionar el control y gerenciamientos de los costos hospitalarios que son excesivamente elevados. Al final si los recursos son insuficientes, sea por la razón que fuere, y las instituciones precisan funcionar con el montante disponible, es preciso saber aplicarlos de forma racional para que todas las unidades de la institución hospitalaria puedan actuar ofreciendo asistencia con mayor padrón de calidad.

En este proceso el enfermero es un elemento indispensable, pues vivencia la asistencia directamente y 24 horas dentro del hospital. Evidentemente todos los profesionales deben estar capacitados para la gerencia de los costos y al mismo tiempo son responsables del uso racional de los recursos y de los gastos en la asistencia.

En el universo pesquisado el proceso de implantación de los centros de costos está todavía en curso. Entretanto, el enfermero precisa comprender que por los factores ya citados, él debe estar capacitado para asumir el papel de gerente de los costos de su unidad cuando este proceso gerencial sea implantado.

La búsqueda de información pautada en el gerenciamiento de los costos y su aprovechamiento en la práctica asistencial y gerencial establece cambios en la cualificación de los profesionales de salud en lo que se refiere, principalmente, a la valorización de los aspectos financieros de la asistencia a la salud y a la comprensión de que la finalidad de gerenciar los aspectos económicos en salud está fundamentada en la optimización de los recursos, en la garantía de acceso y equidad a los usuarios y en el mantenimiento de la calidad de la atención.

\section{REFERENCIAL BIBLIOGRÁFICO}

1- MAXIMIANO, A.C.A. Introdução à administração. 3 ed. São Paulo: Atlas, 1992.

2- BOCCHI, S.C.M.; FÁVERO, N. O processo decisório do enfermeiro no gerenciamento da assistência de enfermagem em um hospital universitário. Separata de: Texto e Contexto Enfermagem, Florianópolis: UFSC, v. 5, n. 2, p. 218-241, jul./dez. 1996

3- FAVERI, F.; FERNANDES, M.S. Função administrativa do enfermeiro: administração da assistência ou administração dos serviços? Separata de: Enfermagem Atual, Rio de Janeiro: EPUB, ano 3, n. 18, p. 32-36, nov./dez. 2003.

4- MOTTA, P.R. Gestão Contemporânea: a ciência e a arte de ser dirigente. 11 ed. Rio de Janeiro: Record, 2002. 
5- KURCGANT, P. et al. Gerenciamento em Enfermagem. Rio de Janeiro: Guanabara Koogan, 2005.

6- ROGANTE,M.M.; PADOVEZE, M.C. Padronização, qualificação e aquisição de materiais e equipamentos médico-hospitalares. São Paulo: EPU, 2005.

7- BRAGA, R. Fundamentos e Técnicas de Administração Financeira. São Paulo: Atlas, 1995.

8- MARTINS, D. Custos e orçamentos hospitalares. São Paulo: Atlas, 2000.

9- CAMPOS, V.F. Controle da qualidade total (no estilo japonês). 6 ed. Belo Horizonte:

Bloch, 1992.

10- MINAYO, M.C.S. O desafio do conhecimento: pesquisa qualitativa em saúde. São Paulo: Hucitec-Abrasco, 1993.

11- LEOPARDI, M.T. Metodologia da Pesquisa na Saúde. Santa Maria: Palloti, 2001. 